\title{
Insuficiência renal aguda por picada de abelhas: relato de casos
}

\author{
Acute renal failure following bee stings: \\ case reports
}

\section{Elizabeth De Francesco Daher ${ }^{1,2}$, Rodrigo Alves de Oliveira ${ }^{3,4}$, Leila Silveira Vieira da Silva ${ }^{3,4}$, Emanuel Maurício Bezerra e Silva ${ }^{4}$ e Talita Peixoto de Morais ${ }^{4}$}

\begin{abstract}
RESUMO
Rabdomiólise é uma síndrome caracterizada por injúria muscular, mais freqüentemente decorrente de esmagamento e traumas musculares. No entanto, a rabdomiólise pode ter também causas não traumáticas, como por exemplo, picadas de abelhas africanizadas. Descrevemos dois casos de rabdomiólise que apresentaram insuficiência renal aguda dialítica após várias picadas de abelhas.
\end{abstract}

Palavras-chaves: Insuficiência renal aguda. Rabdomiólise. Picada de abelhas. Diálise.

\begin{abstract}
Rhabdomyolysis is a syndrome characterized by muscle injury, most frequently due to muscle crushing and trauma. However, it may also be induced by non-traumatic causes, for example by means of stinging by Africanized bees. We describe two cases of rhabdomyolysis that presented dialytic acute renal failure after several bee stings.
\end{abstract}

Key-words: Acute renal failure. Rhabdomyolysis. Bee stings. Dialysis.

As abelhas africanizadas surgiram no Brasil, na década de cinqüenta, originadas de um laboratório de apicultura que realizou a hibridização de abelhas africanas, trazidas da África, com as européias já existentes na natureza brasileira, naquela época. Caracterizam-se por serem muito agressivas, atacando suas vítimas de forma maciça, em enxames, inoculando grande quantidade de veneno ${ }^{2}{ }^{9}$. Nos casos de múltiplas picadas tem sido observada, além dos casos de anafilaxia, a capacidade de causar danos devido ao efeito tóxico direto do veneno, por exemplo insuficiência renal aguda, bem como a morte ${ }^{12791214}$.

A insuficiência renal aguda (IRA) é caracterizada por uma deterioração na função renal que acontece no decorrer de horas a dias, resultando na falência dos rins em excretar produtos nitrogenados e em manter o equilíbrio hidroeletrolítico e ácidobásico $^{1316}$.

A rabdomiólise é uma síndrome caracterizada por lesão muscular extensa com liberação de inúmeras substâncias intracelulares na circulação, podendo ser causada por trauma

\footnotetext{
1. Departamento de Medicina Clínica, Hospital Universitário Walter Cantídio, Faculdade de Medicina, Universidade Federal do Ceará, Fortaleza, CE. 2. Serviço de Nefrologia, Hospital Geral de Fortaleza, Secretaria de Saúde do Estado do Ceará, Fortaleza, CE. 3. Disciplina de Nefrologia, Faculdade de Medicina do Cariri, Universidade Federal do Ceará, Barbalha, CE. 4. Liga Acadêmica de Nefrologia do Cariri, Barbalha, CE. Endereço para correspondência: Prof. Rodrigo Alves de Oliveira. Disciplina de Nefrologia/FMC/UFC. Rua Divino Salvador 284, Rosário, 63180-000 Barbalha, CE. Telefax: 5588 3532-2389/3532-2390

e-mail: rodrigoalves@ufc.br

Recebido em: 09/07/2008

Aceito em 05/03/2009
}

muscular, imobilização prolongada, estado de mal convulsivo, hipertermia maligna, exercício físico extenuante, exposição à drogas e ataques de abelhas africanizadas ${ }^{2311}$.

Em uma revisão de 24 casos de envenenamento por picadas de abelhas, observou-se hemólise e rabdomiólise em 14 e 11 dos casos avaliados, respectivamente ${ }^{7}$. Na literatura, dois relatos de casos de duas crianças picadas cerca de 800 e 500 vezes, respectivamente, evoluíram com rabdomiólise ${ }^{11}$ e IRA ${ }^{8}$ com recuperação da função renal após diálise peritoneal, porém na maioria dos casos a evolução não é satisfatória para adultos com mais de 500 picadas, mesmo na vigência de tratamento dialítico $0^{12712}{ }^{14}$, sendo a mortalidade média nesses casos em torno de $20 \%{ }^{714}$.

Este trabalho foi aprovado pelo Comitê de Ética em Pesquisa da Faculdade de Medicina de Juazeiro do Norte, sob o processo $\mathrm{n}^{\circ} 201$ e tem como objetivo descrever dois casos de acidente por picada de abelhas africanizadas que desenvolveram IRA grave e dialítica secundária a rabdomiólise, com uma evolução fatal e a outra com recuperação total da função renal.

\section{RELATO DOS CASOS}

Caso 1. FTS, sexo masculino, 54 anos, agricultor, foi admitido com história de acidente por múltiplas picadas de abelhas três dias antes do internamento, com lesões distribuídas na face (Figura 1), tronco e membros superiores. Evoluiu com mioglobinúria (Figura 2) seguida de anúria. No exame físico, apresentava-se com estado geral comprometido, 


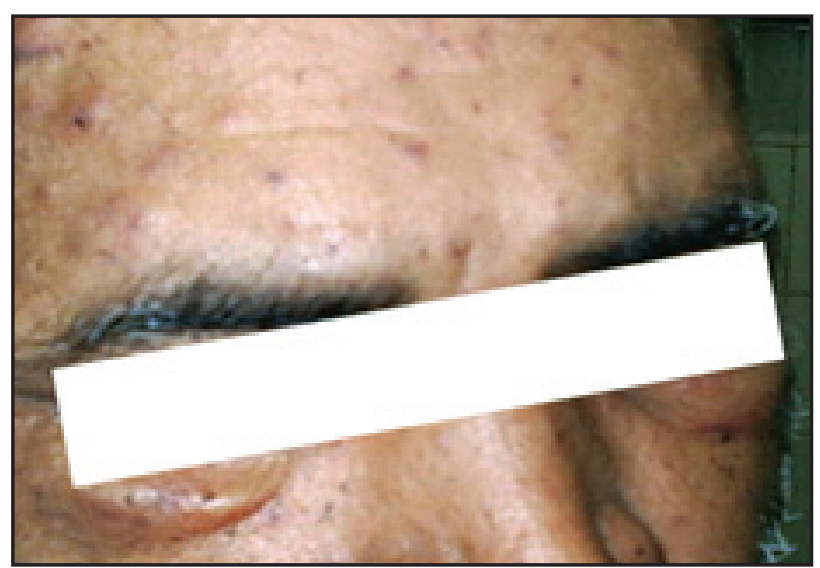

FIGURA 1

Múltiplas picadas de abelha na face do paciente.

torporoso, desorientado, dispnéico, taquicárdico e em anasarca. Ausculta cardiovascular e respiratória normal. Sinais vitais: PA: 160x100mmHg; FC: 126bpm; FR: 24irm; St02: 92\%. Os exames laboratoriais são mostrados na Tabela 1. Tentado estímulo com furosemida $100 \mathrm{mg}$, porém o paciente continuou anúrico, sendo indicado hemodiálise de urgência por síndrome urêmica e hipervolemia. Durante o internamento, realizou várias sessões de hemodiálise, evoluindo com melhora do edema e dos

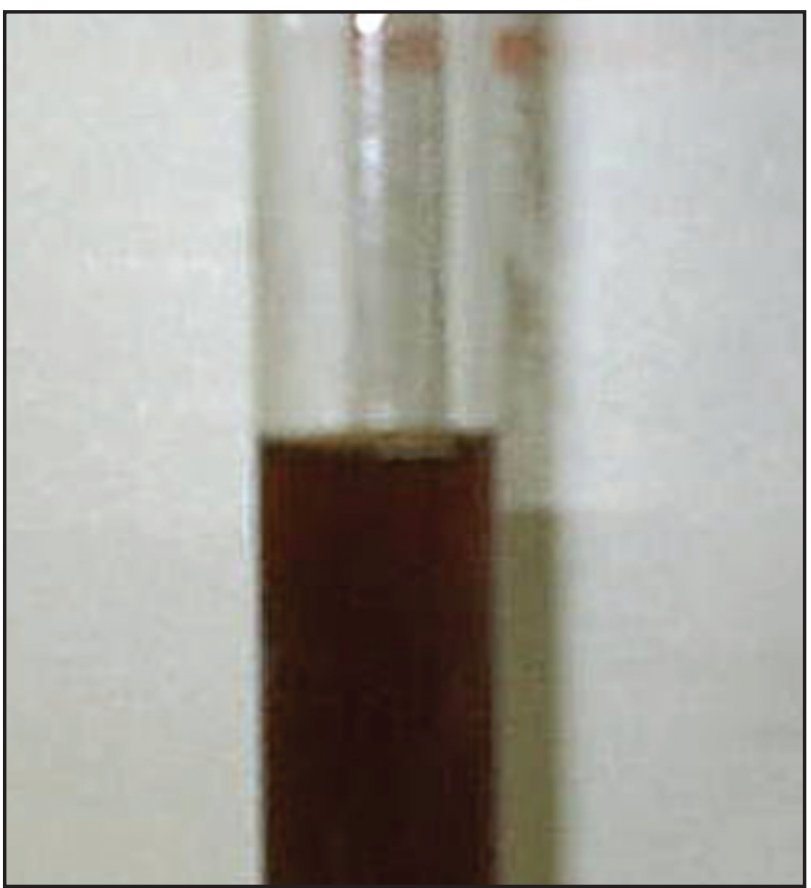

FIGURA 2

Tubo de ensaio com urina, pós-centrifugação. Urina vermelho-escura semelhante a vinho tinto

TABELA 1

Exames laboratoriais do Caso 1.

\begin{tabular}{|c|c|c|c|c|c|c|c|c|}
\hline \multirow[b]{2}{*}{ Exames laboratorias } & \multirow[b]{2}{*}{ Valores normais } & \multicolumn{7}{|c|}{ Dia } \\
\hline & & admissão & 1 & 3 & 18 & 25 & 31 & 38 \\
\hline Uréia $(\mathrm{mg} / \mathrm{dl})$ & $15 \mathrm{a} 40$ & 216 & 118 & 166 & 94 & 119 & 73 & 44 \\
\hline \multirow[t]{2}{*}{ Creatinina $(\mathrm{mg} / \mathrm{dl})$} & (M) 0,5 a 1,0 & 6,3 & 6,12 & 8,86 & 12,6 & 5,9 & 2,2 & 1,4 \\
\hline & (H) 0,7 a 1,2 & & & & & & & \\
\hline Potássio (mEq/l) & 3,5 a 5,0 & 4,3 & 4,7 & 3,9 & 4,2 & & & \\
\hline Sódio $(\mathrm{mEq} / \mathrm{l})$ & 132 a 145 & 120 & 140 & 133 & & & & \\
\hline \multirow[t]{2}{*}{ Hematócrito (\%) } & (M) $36 \mathrm{a} 47$ & 38 & 34,4 & 36 & & & & \\
\hline & (H) 40 a 54 & & & & & & & \\
\hline \multirow[t]{2}{*}{ Hemoglobina $(\mathrm{g} / \mathrm{dl})$} & (M) 11,5 a 16,4 & 12,6 & 11,1 & 11,6 & & & & \\
\hline & (H) 13,5 a 18,0 & & & & & & & \\
\hline Leucócitos totais $\left(\mathrm{mm}^{3}\right)$ & $5.000 \mathrm{a} 10.000$ & 14.500 & 10.900 & 10.200 & & & & \\
\hline Plaquetas $\left(\mathrm{mm}^{3}\right)$ & $150.000 \mathrm{a} 450.000$ & 130.000 & 96.000 & & & & & \\
\hline Glicose (mg/dl) & 69 a 99 & 117 & 145 & 92 & & & & \\
\hline \multirow[t]{2}{*}{$\mathrm{TGO}(\mathrm{U} / \mathrm{l})$} & (M) 10 a 37 & & 414 & & & & & \\
\hline & (H) 11 a 39 & & & & & & & \\
\hline \multirow[t]{2}{*}{ TGP (U/1) } & (M) 10 a 37 & & 167 & & & & & \\
\hline & (H) 11 a 39 & & & & & & & \\
\hline $\mathrm{DHL}(\mathrm{U} / \mathrm{l})$ & 200 a 480 & & 3.124 & & & & & \\
\hline Amilase (U/dL) & 60 a 160 & & 139 & & & & & \\
\hline \multirow[t]{2}{*}{$\mathrm{CK}(\mathrm{U} / \mathrm{l})$} & (M) 24 a 170 & & 22.190 & & & & & \\
\hline & (H) 24 a 195 & & & & & & & \\
\hline Hemoblobina (SU) & negativa & & +++ & & & & & \\
\hline Proteínas( SU) & negativa & & ++++ & & & & & \\
\hline Leucócitos/campo (SU) & negativo & & 11 & & & & & \\
\hline Bactérias (SU) & negativa & & ++ & & & & & \\
\hline
\end{tabular}

TGO: transaminase oxalacética, TGP: transaminase pirúvica, DHL: desidrogenase láctica, CK: creatinofosfoquinase, SU: sumário de urina, M: mulher, H: homem. 
níveis pressóricos, porém persistindo ainda em anúria. Recebeu alta hospitalar no $13^{\circ}$ dia de internamento hospitalar (DIH) para acompanhamento ambulatorial realizando sessões de hemodiálise três vezes por semana. Após 40 dias da admissão, houve aumento progressivo do débito urinário e recuperação da função renal, sendo o tratamento dialítico suspenso.

Caso 2. JFS, sexo masculino, 61 anos, agricultor, chegou ao serviço com quadro de edema generalizado e anúria há um dia. Relatava ter sido vítima de um ataque de abelhas oito dias antes, com múltiplas picadas na face, membros superiores e tronco. No exame físico, apresentava-se com estado geral regular, consciente, desorientado, dispnéico, afebril, hipocorado. Ausculta cardiovascular e respiratória normal. PA: 180x100mmHg. Exame do abdome sem alterações. Os exames laboratoriais encontram-se na Tabela 2. No $3^{\circ} \mathrm{DIH}$, evoluía dispnéico e oligúrico, apesar do uso de furosemida em altas doses. Indicado hemodiálise por uremia e hipervolemia. № $6^{\circ} \mathrm{DIH}$, após três sessões de hemodiálise, ainda se encontrava oligúrico. Evoluiu com picos febris e surgiram pústulas especificamente nos locais das picadas das abelhas, sendo iniciado vancomicina e ceftazidima, empiricamente. Paciente evoluiu com queda do estado geral e hipotensão refratária a volume e droga vasoativa. Evoluiu para o óbito no $8^{\circ} \mathrm{DIH}$.

TABELA 2

\begin{tabular}{|c|c|c|c|c|c|}
\hline \multirow[b]{2}{*}{ Exames laboratoriais } & \multirow[b]{2}{*}{ Valores normais } & \multicolumn{4}{|c|}{ Dia } \\
\hline & & admissão & 1 & 4 & 7 \\
\hline Uréia $(\mathrm{mg} / \mathrm{dl})$ & 15 a 40 & 202 & & 196 & 203,5 \\
\hline \multirow[t]{2}{*}{ Creatinina (mg/dl) } & (M) 0,5 a 1,0 & 15,82 & & 10,67 & 11,32 \\
\hline & (H) 0,7 a 1,2 & & & & \\
\hline Potássio (mEq/l) & 3,5 a 5,0 & 7 & & 5 & 3,8 \\
\hline Sódio (mEq/l) & 132 a 145 & 130 & & & 136 \\
\hline \multirow[t]{2}{*}{ Hematócrito (\%) } & (M) 36 a 47 & 30 & & 26 & 29 \\
\hline & (H) 40 a 54 & & & & \\
\hline \multirow[t]{2}{*}{ Hemoglobina (g/dl) } & (M) 11,5 a 16,4 & 10,1 & & 8,6 & 9,6 \\
\hline & (H) 13,5 a 18,0 & & & & \\
\hline Leucócitos totais $\left(\mathrm{mm}^{3}\right)$ & $5.000 \mathrm{a} 10.000$ & 16.400 & & 30.700 & 24.400 \\
\hline Glicose (mg/dl) & 69 a 99 & 120 & & 144 & 112 \\
\hline \multirow[t]{2}{*}{ TGO (U/l) } & (M) 10 a 37 & 42 & 34 & & \\
\hline & (H) 11 a 39 & & & & \\
\hline \multirow[t]{2}{*}{ TGP (U/l) } & (M) 10 a 37 & 47 & & & \\
\hline & (H) 11 a 39 & & & & \\
\hline DHL (U/l) & 200 a 480 & 387,8 & 352 & & \\
\hline CK (U/l) & 60 a 160 & & 550 & & \\
\hline Hemoglobina (SU) & negativa & +++ & & & \\
\hline Proteínas (SU) & negativa & +++ & & & \\
\hline Leucócitos/campo (SU) & negativo & numerosos & & & \\
\hline Cilindros/campo (SU) & negativo & granulosos & & & \\
\hline Cristais/campo (SU) & negativo & urato amorfo & & & \\
\hline Bactérias (SU) & negativa & + & & & \\
\hline
\end{tabular}

TG0: transaminase oxalacética, TGP: transaminase pirúvica, DHL: desidrogenase láctica, CK: creatinofosfoquinase SU: sumário de urina, M: mulher, H: homem.

\section{DISCUSSÃo}

A insuficiência renal aguda que ocorre após ataques maciços de abelhas é devido a mecanismos tóxicos-isquêmicos com choque hipovolêmico e anafilático associados à lesão tubular por pigmentos devido à lesão muscular (mioglobinúria), hemólise (hemoglobinúria) e/ou necrose tubular aguda (NTA) e devido ao efeito tóxico direto do veneno ${ }^{2911}$. 0 ataque de abelhas pode ser causa de diversas complicações como hipotensão arterial, hemólise, rabdomiólise, distúrbios da coagulação e envolvimento hepático ${ }^{29}$. Apesar da ocorrência de reações alérgicas graves após uma ou poucas picadas, várias mortes tem sido relatadas sem manifestações alérgicas, enfatizando os efeitos tóxicos diretos do envenenamento maciço ${ }^{2910}$. Um estudo demonstrou reações tóxicas sistêmicas após cerca de 50 picadas?.

0 veneno de abelhas é similar entre as diversas subespécies, sendo composto principalmente de melitina, fosfolipase $A_{2}$ e hialuronidase ${ }^{2}$. Os efeitos da toxicidade direta do veneno podem ser divididos em imediatos e tardios. Os sinais e sintomas de reação tóxica imediata são: fadiga, náuseas, vômitos, hemólise, IRA e coagulação intravascular disseminada (CIVD). Hemólise, coagulopatia, trombocitopenia, rabdomiólise, disfunção hepática e CIVD são comumente observadas como manifestações mais tardias, após 12-24 horas do início do quadro ${ }^{410}$.

Nos dois casos relatados, os pacientes tinham menos de 65 anos e evoluíram com anúria, edema e elevação das escórias 
nitrogenadas, apesar do uso de diuréticos, sugerindo a ocorrência de NTA, sendo necessária realização de hemodiálise. Levandose em consideração o profundo impacto da hipovolemia no desenvolvimento da IRA, causada por rabdomiólise, a hidratação venosa agressiva e precoce é uma das medidas terapêuticas mais importantes na abordagem da rabdomiólise, objetivando uma diurese maior que $200-300 \mathrm{ml} /$ hora $^{2611}$. Nos casos relatados, os pacientes chegaram ao serviço com sinais de hipervolemia e uremia, provavelmente já com necrose tubular aguda instalada. A alcalinização da urina pode ser feita para reduzir a formação de agregados de mioglobina dentro dos túbulos, porém está contra-indicada na presença de oligúria com sobrecarga hídrica associada $^{311}$, como nos presentes casos relatados.

Os dois pacientes apresentaram baixos níveis de hemoglobina e hematócrito com elevação da desidrogenase láctica (DHL), sugerindo também ocorrência de hemólise. No primeiro caso, houve diminuição do número de plaquetas e certo grau de lesão hepática, com elevação das transaminases. Trombocitopenia nesses casos é pouco comum, e quando ocorre é devido ao efeito tóxico direto sobre as plaquetas ${ }^{2}$.

Os níveis de creatinofosfoquinase (CK) em ambos os casos estavam elevados, porém no paciente do Caso 2 a elevação não foi tão significativa, provavelmente pelo fato de terem decorrido oito dias do acidente até a chegada ao nosso serviço, mas ainda apresentava mioglobinúria. Os exames laboratoriais não foram realizados diariamente, desta forma não podemos observar a progressão de alguns parâmetros de prognóstico como o nível sérico de CK. Contudo, em alguns casos, a elevação ocasional do nível sérico de CK pode ser o único achado para o diagnóstico presuntivo de rabdomiólise ${ }^{23}$.

No Caso 2, o paciente evoluiu com infecção secundária das lesões de pele, especificamente pústulas nos locais das picadas de abelha, com agente bacteriano não identificado, uma vez que não foi possível coleta de material para realização de cultura e antibiograma, seguido de choque séptico e óbito, apesar do tratamento empírico intensivo com antibiótico, suporte ventilatório e drogas vasoativas. Há relatos de vários casos de morte devido a ataque maciço de abelhas, e as taxas de mortalidade foram calculadas de $15 \%$ a $25 \%$, porém a causa da morte não foi descrita na maioria dos casos $^{2}$. Em um estudo envolvendo pequenos grupos de pacientes, a causa da morte foi insuficiência respiratória ${ }^{27}$. No Caso 1, o paciente recuperou completamente a função renal após um mês de tratamento dialítico.

É muito importante nesses casos a intervenção precoce, com um suporte intensivo adequado, levando em consideração principalmente a hidratação vigorosa e a necessidade de acompanhamento nefrológico com o intuito de reduzir a morbimortalidade da IRA causada por picadas de abelhas. 0 recurso à hemodiálise apresenta vantagens óbvias na rabdomiólise traumática ao permitir a depuração eficiente de produtos tóxicos ${ }^{11}$.
Outro tópico importante que devemos levar em consideração é a remoção precoce dos ferrões. Estudo realizado no Departamento de Entomologia da Universidade da Califórnia, EUA, evidenciou que quanto mais tempo se passa para a remoção dos ferrões nas vítimas de picadas de abelhas, mais veneno é inoculado nestas, não havendo diferença significativa na forma de remoção, quer seja por pinçamento, quer seja por raspagem. Não é mencionado sobre o tempo máximo para esta remoção, apenas enfatizam que a mesma deve ocorrer o mais rápido possível ${ }^{15}$.

Conclui-se que a IRA por rabdomiólise induzida, por picadas de abelhas africanizadas, é grave e associada a uma elevada mortalidade, devendo ser tratada precocemente e de forma agressiva.

\section{REFERÊNCIAS}

1. Bresolin NL, Carvalho LC, Goes EC, Fernandes R, Barotto AM. Acute renal failure following massive attack by Africanized bee stings. Pediatric Nephrology 17: 625-627, 2002.

2. Daher EF, Silva Junior GB, Bezerra GP, Pontes LB, Martins AMC, Guimarães JA. Acute renal failure after massive honeybee stings. Revista do Instituto de Medicina Tropical de São Paulo 45: 45-50, 2003.

3. Daher EF, Silva Junior GB, Brunetta DM, Pontes LB, Bezerra GP. Rhabdomyolysis and acute renal failure after strenuous exercise and alcohol abuse: case report and literature review. Sao Paulo Medical Journal 123: 33-37, 2005.

4. Fighera R, Souza T, Barros C. Acidente provocado por picada de abelhas como causa de morte de cães. Ciência Rural 37: 590-593, 2007.

5. Habermann E. Bee and wasp venoms. Science 177: 314-322, 1972.

6. Liãno F, Junco E, Pascual J, Madero R, Verde E, Madri Acute Renal Failure Study Group. The spectrum of acute renal failure in the intensive care unit compared with that seem in other settings. Kidney International 53(supl 66): S16-S24, 1998.

7. Mejia G, Arbelaez M, Henao JE, Sus AA, Arango JL. Acute renal failure due to multiple stings by africanized bees. Annals of Internal Medicine 104: 210-211, 1986.

8. Moraes Filho M, Lima JCA, Dantas MM. Insuficiência renal aguda. In: Filgueira N, Costa I, Cordeiro C, Lucena V, Ramos H, Brito C (eds) Condutas em clínica médica, $2^{\text {a }}$ edição, Editora Medsi, Rio de Janeiro, p.483-490, 2001.

9. Oliveira FA, Guimarães JV, Reis MA, Teixeira VPA. Acidente humano por picadas de abelhas africanizadas. Revista da Sociedade Brasileira de Medicina Tropical 33: 403-405, 2000.

10. Penteado JOP, Oliveira CH, D’Angieri A, Graudenz GS, Massucato AE, Castro AB, Castro ESV. 3200 picadas de abelhas africanizadas. Revista Brasileira de Terapia Intensiva 15: 176-179, 2003.

11. Rosa NG, Silva G, Teixeira A, Rodrigues F, Araújo JA. Rabdomiólise. Acta Médica Portuguesa 18: 271-282, 2005.

12. Schumacher MJ, Egen NB. Significance of africanized bees for public health: a review. Archives of Internal Medicine 155: 2038-2043, 1995.

13. Thadhani R, Pascual M, Bonventre JV. Acute renal failure. New England Journal of Medicine 334: 1448-1460, 1996.

14. Thiruventhiran T, Goh BL, Leong CL, Cheoh PL, Looi LM, Tan SY. Acute renal failure following multiple wasp stings. Nephrology Dialysis Transplantation 14: 214-217, 1999.

15. Visscher PK, Vetter RS, Camazine S. Removing bee stings. Lancet 348: 301-302, 1996.

16. Yu L, Santos BF, Burdmann EA, Suassuna JHR, Batista PBP. Insuficiência renal aguda. Diretrizes da Sociedade Brasileira de Nefrologia/Comitê de Insuficiência Renal Aguda da Sociedade Brasileira de Nefrologia, 2007. 